\title{
BMJ Open An investigation of routes to cancer diagnosis in 10 international jurisdictions, as part of the International Cancer Benchmarking Partnership: survey development and implementation
}

David Weller, ${ }^{1}$ Peter Vedsted, ${ }^{2}$ Chantelle Anandan, ${ }^{1}$ Alina Zalounina, ${ }^{2}$ Evangelia Ourania Fourkala, ${ }^{3}$ Rakshit Desai, ${ }^{3}$ William Liston, ${ }^{3}$ Henry Jensen, ${ }^{2}$ Andriana Barisic, ${ }^{4}$ Anna Gavin, ${ }^{5}$ Eva Grunfeld, ${ }^{6}$ Mats Lambe, ${ }^{7}$ Rebecca-Jane Law, ${ }^{8}$ Martin Malmberg, ${ }^{9}$ Richard D Neal, ${ }^{8}$ Jatinderpal Kalsi, ${ }^{3}$ Donna Turner, ${ }^{10}$ Victoria White, ${ }^{11}$ Martine Bomb, ${ }^{12}$ Usha Menon, ${ }^{3}$ ICBP Module 4 Working Group*

To cite: Weller D, Vedsted $P$, Anandan $\mathrm{C}$, et al. An investigation of routes to cancer diagnosis in 10 international jurisdictions, as part of the International Cancer Benchmarking Partnership: survey development and implementation. BMJ Open 2016;6:e009641. doi:10.1136/bmjopen-2015009641

- Prepublication history and additional material is available. To view please visit the journal (http://dx.doi.org/ 10.1136/bmjopen-2015009641).

Received 7 August 2015 Revised 11 May 2016 Accepted 24 May 2016

CrossMark

For numbered affiliations see end of article.

Correspondence to Professor David Weller; david.weller@ed.ac.uk

\section{ABSTRACT}

Objectives: This paper describes the methods used in the International Cancer Benchmarking Partnership Module 4 Survey (ICBPM4) which examines time intervals and routes to cancer diagnosis in 10 jurisdictions. We present the study design with defining and measuring time intervals, identifying patients with cancer, questionnaire development, data management and analyses.

Design and setting: Recruitment of participants to the ICBPM4 survey is based on cancer registries in each jurisdiction. Questionnaires draw on previous instruments and have been through a process of cognitive testing and piloting in three jurisdictions followed by standardised translation and adaptation. Data analysis focuses on comparing differences in time intervals and routes to diagnosis in the jurisdictions.

Participants: Our target is 200 patients with symptomatic breast, lung, colorectal and ovarian cancer in each jurisdiction. Patients are approached directly or via their primary care physician (PCP). Patients' PCPs and cancer treatment specialists (CTSs) are surveyed, and 'data rules' are applied to combine and reconcile conflicting information. Where CTS information is unavailable, audit information is sought from treatment records and databases.

Main outcomes: Reliability testing of the patient questionnaire showed that agreement was complete $(\kappa=1)$ in four items and substantial $(\kappa=0.8,95 \% \mathrm{Cl}$ 0.333 to 1 ) in one item. The identification of eligible patients is sufficient to meet the targets for breast, lung and colorectal cancer. Initial patient and PCP survey response rates from the UK and Sweden are comparable with similar published surveys. Data collection was completed in early 2016 for all cancer types.

\section{Strengths and limitations of this study}

- There are no previous examples of applying standardised survey methods in a broad range of jurisdictions to examine components of diagnostic intervals in cancer.

- This study could provide unique insights into why cancer survival differences exist in different regions of the world.

- International Cancer Benchmarking Partnership Module 4 Survey is a questionnaire-based survey, and results are subject to limitations and caveats-particularly those arising from nonresponse bias and external validity.

- Response to a questionnaire on diagnostic journeys in patients with cancer may be influenced by factors which differ across jurisdictions, compounding existing non-response bias.

Conclusion: An international questionnaire-based survey of patients with cancer, PCPs and CTSs has been developed and launched in 10 jurisdictions. ICBPM4 will help to further understand international differences in cancer survival by comparing time intervals and routes to cancer diagnosis.

\section{BACKGROUND}

The International Cancer Benchmarking Partnership (ICBP) is a major international collaboration which is exploring differences in cancer survival between Australia, Canada, Denmark, Norway, Sweden and the UK. ${ }^{1}$ It has 
already demonstrated significant differences in 1-year and 5-year relative survival for breast, lung, colorectal and ovarian cancers among participating jurisdictions. ${ }^{2}$ Recent data confirm that significant disparities in cancer survival persist across a range of tumour types in Europe. ${ }^{3}$ The ICBP further seeks to systematically explore population and healthcare-related factors in relation to these variations in cancer survival. Survival differences between populations are most probably due to a range of factors including lifestyle, levels of comorbidity, availability of screening programmes, primary care system, and availability and quality of diagnostic and treatment services. $^{2}{ }^{4-7}$ Module 4 of the ICBP (ICBPM4) aims to:

- Compare time intervals (patient, primary care, diagnostic and treatment and total intervals-see figure 1) between jurisdictions.

- Identify the proportion of patients entering the cancer pathway through different routes (eg, symptom-based diagnosis, screening, via accident and emergency (A\&E)) and analyse the association with time intervals within jurisdictions.

- Analyse the association between time intervals (outlined in the methods section) and cancer outcomes in participating jurisdictions and identify where actions to reduce delays could be focused.

The central research question of ICBPM4 is: 'can variations in cancer survival between jurisdictions participating in the International Cancer Benchmarking Partnership be explained by differences in routes to diagnosis?' Routes to diagnosis have an important influence on cancer outcomes and patient experience. For common cancers, such as colorectal cancer, available evidence suggests that longer patient intervals (the time from first noticing symptoms to seeking help) are associated with poorer survival, ${ }^{8}$ and that longer diagnostic intervals (from first presentation until diagnosis) increase mortality. ${ }^{9-12}$ There is a growing body of evidence examining the relationship between various components of the diagnostic journey and long-term cancer outcomes; emergency presentation is typically associated with worse patient outcomes, and this negative effect persists after adjustment for stage ${ }^{13}$ Further, while their number of prediagnosis consultations has not been quantified, qualitative evidence suggests that patients who present as emergencies typically have prolonged, circuitous pathways, in which consultations are not necessarily a conduit to diagnosis. ${ }^{14}$ There is some observational evidence that prolonged primary care time intervals can adversely affect outcomes, and similar associations are found with longer treatment intervals, ${ }^{15}$ although evidence across a range of cancers is mixed. ${ }^{12}$ In terms of patient experience, it is known that patients prioritise rapid investigation for cancer, ${ }^{16}$ and 'time to diagnosis' affects patients' confidence in the healthcare system. ${ }^{17}$ Hence, investigating differences in routes to cancer diagnosis between different countries can help in assessing the possible impact on cancer survival.
Accordingly, there is a great deal of interest in shortening diagnostic intervals through better access to investigations, and decision aids in primary care, ${ }^{18}{ }^{19}$ as there are some indications that variation in access, and readiness to use, diagnostic investigations might influence cancer outcomes; ${ }^{60}$ the gatekeeping function in primary care may also influence diagnostic intervals. ${ }^{21}$ Longer diagnostic intervals may lead to cancers which are more advanced at the time of diagnosis; there is some evidence, for example, of later stage diagnoses in the UK compared to other countries, notably for lung and colorectal cancers. ${ }^{22}{ }^{23}$ Further, treatment intervals have been shown to be associated with stage progression. ${ }^{24} 25$

ICBP Module 1 showed that cancer survival is higher in Sweden, Canada and Australia, intermediate in Norway and lower in Denmark and the UK. ${ }^{2}$ Further analysis in Module 1 suggests that differences in treatment and access to optimal treatment may be impacting on survival outcomes, alongside evident 'delays' in diagnosis. ${ }^{46}$ ICBP Module 2 demonstrated that awareness and beliefs about cancer are unlikely to explain international survival differences but may form part of a more complex picture. ${ }^{5}$ ICBP Module 3 compared primary care systems and found differences in primary care physician (PCP) readiness to investigate between countries that could be related to cancer outcomes. ${ }^{6}$ Given the international differences in survival and the scientific basis for the importance of routes to cancer diagnosis and treatment, a detailed examination of international differences in diagnostic pathways is important.

The purpose of this paper is to present the study design (highlighting key issues in defining and measuring milestones and time intervals), and to report on processes of identifying patients with cancer, questionnaire development, data management and analyses.

\section{Management of ICBP}

The ICBP is overseen by a Programme Board with representatives from all participating jurisdictions. The Board meets regularly to review progress and findings from each of the modules. Dedicated module chairs, overseeing each module, report to the Programme Board. ICBPM4 is led by a central academic team with module co-chairs and senior researchers based at University College London (UCL), UK, the University of Edinburgh, UK and Aarhus University, Denmark. Cancer Research UK provides programme management support to the central team. ${ }^{27}$ The ICBPM4 central academic team and the overall programme management are funded jointly by participating jurisdictions. Each of the ICBPM4 jurisdictions has an individual lead and management team to conduct their own local survey. Regular meetings take place between the jurisdiction leads and the central academic team.

\section{ICBPM4 study design}

The study is an international, multicentre, crosssectional, population-based survey of newly diagnosed patients with cancer. Ten jurisdictions (Australia (Victoria), 
Canada (Manitoba, Ontario), Denmark, Norway, Sweden and the UK (England, Northern Ireland, Scotland and Wales)) are participating. These jurisdictions were selected as they are considered to be reasonably similar regarding access and expenditure on healthcare and level of cancer treatment but show variation in cancer survival. They all have comprehensive cancer registration facilitating international studies. ${ }^{1}$

In common with other ICBP modules, ${ }^{2}$ four cancers are included-breast, ovarian, colorectal and lung cancer. There is variation among these cancers in terms of their symptom characteristics, availability of screening, treatments and effects of other factors like age and comorbidity. For a given period in each jurisdiction, newly diagnosed patients with cancer were identified and a questionnaire was sent to the patient, the PCP and the diagnosing/treating hospital. The questionnaire focused particularly on specific milestones, time intervals and the routes to diagnosis. The study was initiated in 2009 and the data collection launched in 2013. Data collection finished in early 2016.

\section{Routes to diagnosis}

An understanding of differences in routes to diagnosis between jurisdictions is important to understand differences in time intervals and where, if feasible, to focus an intervention. Cancers may be diagnosed through routes other than symptomatic presentation to primary care, such as screening or direct presentation to a specialist or hospital emergency department. The survey sought to capture this variety of diagnostic routes.

Nevertheless, it is difficult to capture complex patient journeys in surveys; the questionnaire drew on previous 'routes to diagnosis' research ${ }^{13}$ but we refined previous definitions of time points and intervals, using guidance from the Aarhus Statement. ${ }^{28}$ Further, we specified routes to be explored as: (1) symptoms/a bodily change prompting a doctor's visit; (2) symptoms/a bodily change prompting a visit to accident and emergency
(A\&E); (3) combinations of (1) and (2); (4) incidental diagnosis in the course of investigation or treatment for another problem; (5) participation in a populationbased cancer screening programme; (6) other route. Using these predefined routes, it was possible to standardise data between jurisdictions-it also made our classifications consistent with those used in ICBP Module 3, enabling the combination of data with these modules in future analyses. ${ }^{6}$

\section{Measuring time points and intervals}

A central aim of ICBPM4 is to measure and compare time intervals (patient, primary care, diagnostic and treatment intervals) in participating jurisdictions. To build on the best existing knowledge and conceptualisation of time intervals, we used the Aarhus Statement ${ }^{28}$ (see figure 1) and focused on four key time points, defined as shown in table 1 . We sought to define, as precisely as possible, the different time intervals, as measurement of time points can all present methodological challenges-it is also necessary to decide which source of data (patient, PCP, CTS) is preferred for each of the time points, in order to generate data rules when there are multiple sources of information:

- Date of first symptom-cancer symptoms can be multiple, vague and non-specific, making it difficult to precisely identify this date (collected, in ICBPM4, from patient and PCP. Preferred reporting source: patient).

- Date of first presentation to primary care-similarly, it is often difficult to establish which 'presentation' in primary care represents the first time a patient seeks help about cancer-related symptoms (collected from patient and PCP. Preferred reporting source: PCP).

- Date of referral-there can be confusion over the date on which the PCP engages specialist diagnostic and treatment services for the patient's ongoing management; in this study, we defined date of first referral as the date where the PCP transferred the
Figure 1 Key time points and diagnostic intervals in the route from first symptom until start of treatment. $^{28}{ }^{36} \mathrm{PCP}$, primary care physician.

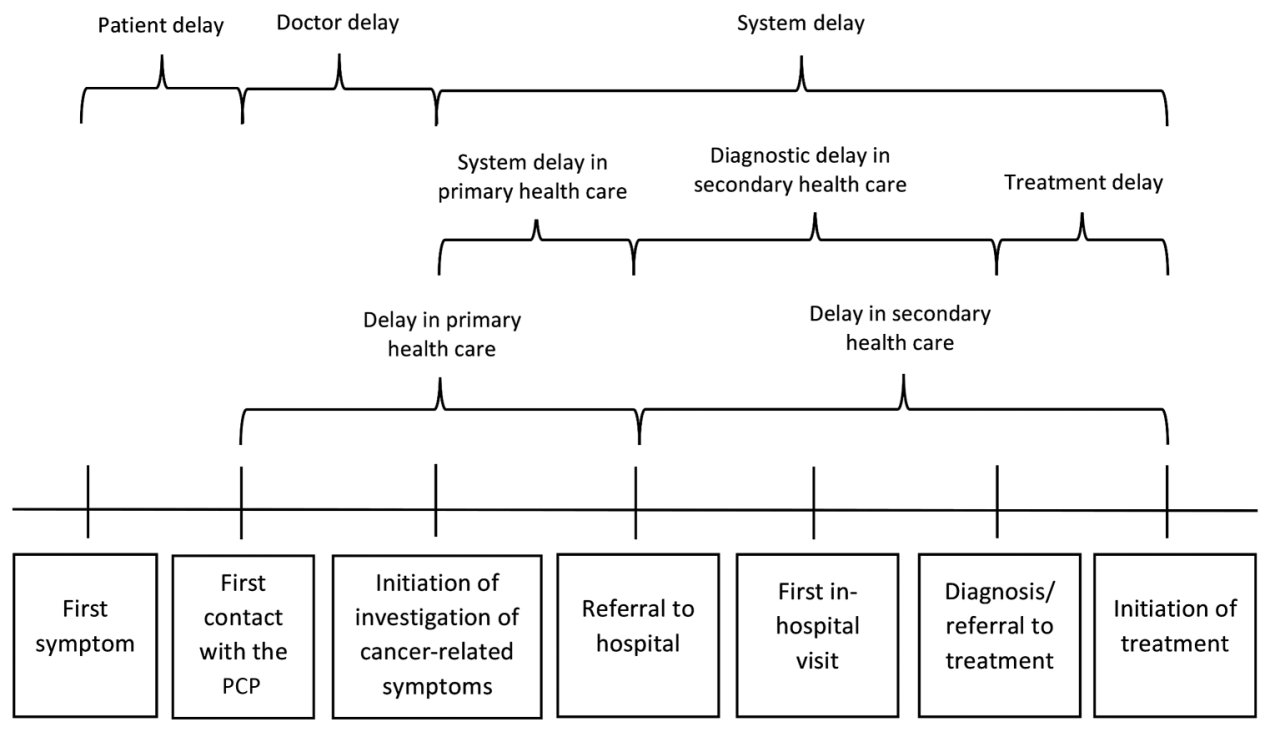


Table 1 Time point definitions based on the 'Aarhus Statement' 28

Date of first symptom The time point when first bodily changes and/or symptoms are noticed. Should encompass several key components: the date when the first bodily change was noticed, the date when the first symptom was noticed, the date when the person perceives a reason to discuss the symptom with a healthcare professional and the date when the first 'alarm' or 'high-risk' symptom was noticed.

Date of first

presentation

The time point at which, given the presenting signs, symptoms, history and other risk factors, it would be at least possible for the clinician seeing the patient to have started investigation or referral for possible important pathology, including cancer.

Date of referral

The time point at which there is a transfer of responsibility from one healthcare provider to another (typically, in 'gatekeeper' healthcare systems, from a primary care provider to a doctor/service specialising in cancer diagnosis and management) for further clinical diagnostic and management activity, relating to the patient's suspected cancer. Patients may be referred more than once or between specialists.

Date of diagnosis

Studies should be explicit about how the date is measured, and should consult the well-developed hierarchical rationales available in the public domain in choosing their definition of date of diagnosis.

responsibility for further diagnosis and treatment to specialist services (collected from PCP).

- Date of diagnosis-a range of definitions exist, including dates of (1) tissue diagnosis and (2) receiving results of investigations. Therefore, respondents were asked to report date of diagnosis and, additionally, what they based their understanding of date of diagnosis on (collected from patient, PCP and CTS. Preferred reporting source: CTS $>$ PCP $>$ patient)

Examples of questions we used to gather information about these time points (from the patient with breast cancer questionnaire) are shown in table 2. Further complexity arises from the nature of diagnostic pathways; patients do not typically follow a linear pattern from the onset of symptoms to presentation in primary care to diagnosis and treatment; sometimes there will be cross referrals; other patients will be returned to primary care only to be referred to a different service. Hence, while this research seeks to measure discrete intervals, there is recognition that time points and intervals can be difficult to identify and measure accurately.

\section{Health-related visits and investigations}

In addition to time points and intervals, ICBPM4 seeks information on the volume of visits to healthcare providers and diagnostic facilities amongst patient respondents. Hence, we included, in the patient survey, questions on types (and number) of health-related visits in the lead up to patients' cancer diagnosis.

\section{Questionnaire development and pilot testing}

For data collection, we chose a questionnaire-based survey (complemented by registry data where appropriate and possible). While interviews might provide an opportunity to better capture patient journey complexity ${ }^{29}$ this would have been difficult to standardise across 10 jurisdictions, given translation and local adaptation issues. This method also allowed us to include significant numbers of patients with cancer and enhance statistical precision.
To be able to triangulate and supplement reports of time points and intervals, we chose a strategy where we, for each individual patient, survey the patient with cancer, the patient's PCP and the CTS. Reports of diagnostic pathways can vary between patients and their healthcare providers $;{ }^{30-32}$ our approach takes account of this variation. Accordingly, the data collection comprises three surveys per cancer:

- Patients with cancer: own experiences of symptoms are vital in determining the onset of symptoms, how long it took to seek help and consultations/investigations along the path to diagnosis.

- Patients' PCPs: are an important source of data on the cancer diagnostic pathway ${ }^{31}$ as most patients present to primary care with symptoms and primary care records are a good source of information on, for example, comorbidity.

- Patients' CTSs: are a particularly important source of diagnostic and treatment information.

\section{Questionnaire item generation}

We developed a core questionnaire based on the predefined list of required variables. This core questionnaire was then developed further for each of the four cancers in order to adjust for cancer-specific issues. While guidance on measuring time points and intervals in the cancer patient pathway is available, ${ }^{28}$ until now there is no widely accepted, validated instrument for use in such surveys. We generated a series of questions from existing instruments in Denmark and the UK, ${ }^{32} 33$ and complemented this with an item generation exercise involving senior members of the ICBP team. Wording of the questions reflected a balance between providing enough detail to address the complexity of patient journeys, avoiding an overly burdensome instrument and getting a standardised description. To allow for characterisation and comparison of patients with cancer between countries and different groups, we included items on potential confounders, including comorbidity, smoking, age, gender and educational level. 
Table 2 Areas of enquiry, number of items and extracts of questions used to elicit time points (example from breast cancer questionnaires)

\begin{tabular}{|c|c|c|}
\hline $\begin{array}{l}\text { Patient } \\
\text { Background (1) } \\
\text { Route to diagnosis } \\
\text { (eg, via PCP, A\&E) (1) } \\
\text { Description of symptoms and date first } \\
\text { noticed (2) } \\
\text { Time taken to consult doctor (1) } \\
\text { Time to get an appointment and date } \\
\text { seen (2) } \\
\text { Number of health professional visits (1) } \\
\text { Time taken to get CTS appointment (2) } \\
\text { Date of diagnosis (1) } \\
\text { Description of treatments received (1) } \\
\text { Details of CTS (1) } \\
\text { General health and comorbidity (2) } \\
\text { Sociodemographics (3) }\end{array}$ & $\begin{array}{l}\text { PCP } \\
\text { Duration of symptoms prior to } \\
\text { presentation (1) } \\
\text { Route to diagnosis (1) } \\
\text { Investigations ordered and dates (1) } \\
\text { Date of referral to CTS, and details of } \\
\text { referral (3) } \\
\text { Date of diagnosis (1) } \\
\text { - Comorbidity information (1) }\end{array}$ & $\begin{array}{l}\text { CTS } \\
\text { Date of first attendance for } \\
\text { specialist services (1) } \\
\text { Route of referral (1) } \\
\text { - Where patient seen (1) } \\
\text { Date of diagnosis (1) } \\
\text { Date cancer treatment started (1) } \\
\text { - Tumour information (2) }\end{array}$ \\
\hline \multicolumn{3}{|c|}{$\begin{array}{l}\text { Please write down your best estimate of the date you noticed ... any symptom(s) you may have had before contacting a } \\
\text { doctor or taking part in screening }\end{array}$} \\
\hline \multicolumn{3}{|c|}{ Date of first presentation to primary care (patient questionnaire) } \\
\hline \multirow{2}{*}{\multicolumn{3}{|c|}{$\begin{array}{l}\text { What was the date you first saw your doctor about your health concern(s) or symptom(s)? } \\
\text { Date of first presentation to primary care (PCP questionnaire) }\end{array}$}} \\
\hline & & \\
\hline \multicolumn{3}{|c|}{$\begin{array}{l}\text { Through what route did the patient first present? (if your patient first presented to prima } \\
\text { Can you please provide your best approximation of the date of his primary care visit? } \\
\text { Date of referral (PCP questionnaire) }\end{array}$} \\
\hline \multicolumn{3}{|c|}{$\begin{array}{l}\text { At what date did you first refer the patient to hospital or another specialist, thereby transferring the responsibility for ongoing } \\
\text { investigation/treatment to other medical services? }\end{array}$} \\
\hline \multicolumn{3}{|c|}{ Date of diagnosis (patient questionnaire) } \\
\hline \multirow{2}{*}{\multicolumn{3}{|c|}{ What was the date you were told you had cancer? }} \\
\hline & & \\
\hline \multicolumn{3}{|c|}{$\begin{array}{l}\text { Please indicate date of diagnosis: This can be decided in different ways; please tick and complete as many of the following } \\
\text { dates as possible: Date of histological confirmation; date of results of investigation confirming cancer;date patient was told; } \\
\text { date of biopsy; date patient was first admitted to hospital because of themalignancy;date of MDT confirmation of diagnosis; } \\
\text { other (please specify) }\end{array}$} \\
\hline
\end{tabular}

Based on this work, three core questionnaires for each cancer were developed in English (a total of 12 questionnaires). Jurisdictions were able to add a small number of locally relevant questions to the core questionnaire-these are not included in the core analyses.

\section{Cognitive testing of draft instrument}

Based on established principles of questionnaire development, ${ }^{34}$ the questionnaires underwent a process of cognitive testing, exploring the understanding of items and use of the responses. We also sought feedback on any ambiguous question wording, layout and order.

Cognitive testing was undertaken in England and Scotland from February to May 2012. Patients were provided with the draft questions in advance, and then asked to provide feedback via focus groups, telephone, face-to-face interviews and email. A total of 14 patients took part in this exercise; in England, patients were recruited opportunistically while attending a gynaecological clinic, while in Scotland they were recruited through a general practice in Edinburgh.

Five PCPs and eight CTSs in the UK also provided feedback on the PCP and CTS versions of the questionnaire. Beside participants' overall assessment of the questionnaire, participants were asked for each item:

- Is the meaning of this question, and why we are asking it, clear?

- Did you have any trouble answering this question?

- Do you have any suggestions on how this question could be improved?

As a result of cognitive testing, wording of several items was modified, with the aim of achieving greater clarity and ease of completion. The questionnaires were revised and reformatted to produce a version for piloting.

\section{Pilot-testing}

This was undertaken in three jurisdictions-England, Northern Ireland and Victoria. The pilot was designed to (1) test identification and recruitment procedures 


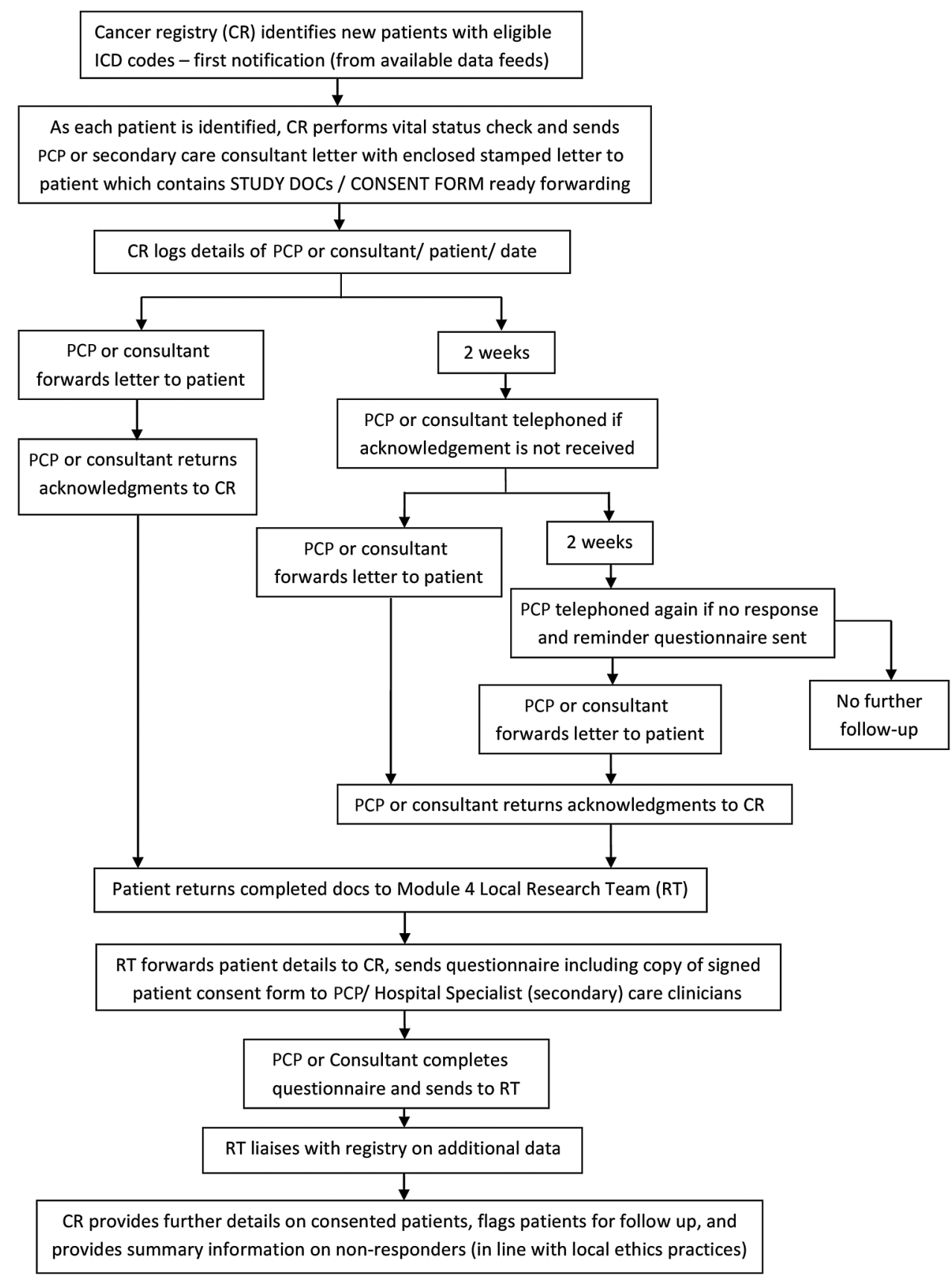

Figure 2 Patient identification and data collection process for pilot in England (also documented in main study). PCP, primary care physician.

(see figure 2), and (2) seek further feedback on the questionnaire and other study materials.

The pilot examined the key trial processes illustrated in figure 2 including vital status checks (to ensure all identified patients were alive before contacting these patients' individual PCP) and of PCP and patient awareness of the cancer diagnosis. It also examined the process of PCP forwarding of patient questionnaires (an ethical requirement in England, Scotland, Wales and Norway) and questionnaire completion rates.

The majority of pilot patients were in England where patients were recruited from three cancer registries (as they existed at the time-Trent Cancer Registry, the
Eastern Cancer Registration and Information Centre and the West Midlands Cancer Intelligence Unit became part of a single English registry during the course of our study). Two hundred consecutive patients (50 per cancer) were identified, of whom $144(72 \%)$ were female. After application of eligibility criteria and PCP identification, 120 were sent to PCPs for forwarding to patients.

PCPs forwarded 72 of the 120 questionnaires (59.7\%) to patients. The most common reported reasons for not forwarding were 'patient deceased' or 'change of address'. Forty-three completed questionnaires were received $(36 \%)$; of these, $39(90.7 \%)$ provided consent 
to have their PCPs and CTSs contacted. The response rate for PCP and CTS surveys was $87 \%$ and 58\%, respectively.

Findings from all three regions of the pilot were broadly similar, and highlighted the need to improve recruitment and response rates through a range of strategies:

- Improvement in questionnaire follow-up procedures.

- Change to patient recruitment period from 26 months postdiagnosis to 3-6 months after diagnosis to minimise numbers of patients still undergoing active treatment and provide additional time for patient's cancer registrations to be validated.

- Reduction of complexity and burden of the questionnaires-through improved formatting, clearer response categories, more logical progression through the questionnaires and removal of lengthy items (such as those on comorbidity).

\section{Reliability testing}

Reliability is an important issue when measuring dates and information exposed to recall bias. To improve reliability, we used items that had been tested previously. Further, 12 patients agreed to complete a second questionnaire after 2 weeks for a test-retest. Questions with minimum 10 patients' responses were included in the analysis. The agreement for categorical variables was assessed by $\kappa$ coefficients. The CIs for $\kappa$ were biascorrected using bootstrapping. The agreement for date of diagnosis was assessed by a description of the variation in the differences between the dates. Among the five categorical variables considered, the agreement was substantial $(\kappa=0.8,95 \%$ CI 0.333 to 1$)$ for one and perfect $(\kappa=1,95 \%$ CI NA) in four (owing to the complete agreement, the CI could not be calculated using bootstrapping; the statistical precision might still, due to the ambiguous assumptions, be difficult to establish). For date of diagnosis, $54.5 \%$ of the responders reported the same date, while for $18.2 \%$ and $27.3 \%$ the difference was 1-8 days and more than 8 days, respectively.

\section{Translation and cultural adaptation of the questionnaires}

In Wales, while the questionnaire itself was not translated, the study summary and covering letter were translated into Welsh. Translated versions were required for Sweden, Denmark and Norway. To make the process efficient, the Scandinavian languages were serially translated. Once a Danish translated and culturally adapted version of the questionnaire was produced, it was translated and culturally adapted for use in Sweden and Norway. The Scandinavian translation was undertaken as follows:

- Questionnaires were translated from English into Danish by external translators with Danish as their mother tongue.

- Translations were double checked for wording by another independent highly English linguistic skilled source with Danish as first language.
- The two versions were synthesised and problems discussed and resolved as follows:

- All questions with no discrepancies between translations-accepted.

- Questions with minor discrepancies in translations -wording changed according to second linguistic source by project group.

- Questions with other than minor discrepancieschanged by consensus by international project group and linguistic worker.

The final version was then translated from Danish into Swedish and Norwegian with no back-translation. If there were any questions and problems with the Scandinavian translations, they were discussed and solved at telephone meetings.

Field-testing and cultural adaptation in Englishspeaking jurisdictions outside the UK was undertaken to ensure that cultural, social and other contextual issues were solved. In particular, differences in terminology exist for some health-related words and phrases between the countries. Further, differences in the structure of health services between jurisdictions meant that terms such as 'referral' or 'primary care' needed cultural adaptation.

At the completion of translation and local adaptation processes, a final check was made by the central ICBP team and jurisdiction leads to ensure consistency in meaning and interpretation of the questionnaires across jurisdictions. A summary of questionnaire items is shown in table 2. The three questionnaires for each of the four cancers are supplied (see online supplementary files S1S3). Researchers should get in touch with the ICBP programme management team at icbp@cancer.org.uk prior to referencing or using the ICBPM4 questionnaires.

\section{Patient identification and recruitment}

In each jurisdiction, the aim is to collect information from 200 symptomatic patients and their associated PCPs and CTSs for each of the four cancers. Since data from patients whose cancers were screen-detected do not contribute information about all relevant time points and intervals, efforts are being made to recruit 200 patients whose cancers were detected via a symptomatic route, for each cancer in each jurisdiction. Expected recruitment rates are lowest for ovarian cancer, and in Sweden ovarian cancer is not included. Eligible participants are consecutive, newly diagnosed patients with cancer diagnosed 3-6 months prior to identification through the cancer registry. Inclusion and exclusion criteria are shown in box 1.

All participating jurisdictions have comprehensive and valid cancer registries, based on internationally accepted criteria. ${ }^{35}$ Hence, we are able to undertake registry-based recruitment of patients; while registries differ in their capacity to identify patients in a timely manner, ${ }^{36}$ they are the least likely of all patient identification methods to produce sampling bias. ${ }^{37}$ There are minor differences in recruitment approaches between jurisdictions, for example, Northern Ireland verifies patient eligibility 
Box 1 Inclusion and exclusion criteria for the four cancer types in International Cancer Benchmarking Partnership Module 4

\section{Inclusion criteria:}

1. Patients aged 40 years or more.

2. Diagnosis of primary (behaviour code 3 on the third revision of the International Classification of Diseases for Oncology; ICD-0-3) cancers with the following ICD10 codes

A. Breast: (C50.0-C50.9)

B. Ovary including the fallopian tube and adnexa (C56 (ICD-0-3 C56.9); C57.0-C57.9)

C. Lung and bronchus: (C34.0-C34.9)

D. Colorectal: colon (ICD-10 C18.0-C18.9), rectosigmoid junction (C19; ICD-0-3 C19.9) and rectum (C20; ICD-0-3 C20.9)

3. All patients with a qualifying index cancer were included, irrespective of whether it was their first, second or higher-order cancer

4. Completion of patient questionnaire within 6 months of cancer diagnosis

Exclusion criteria:

1. Patients where the tumour was benign (behaviour code 0 ), of uncertain or borderline malignancy (behaviour code 1), in situ (behaviour code 2) or metastatic to the index organ from elsewhere (behaviour code 6).

2. Patients who had previously had a separate cancer of the same organ (eg, consecutive breast cancers)

3. Patients who had cancers of the anus and anal canal (ICD-10 C21) and trachea (C33)

4. Male patients with breast cancer (C50)

5. Patients who have synchronous primary cancers

through checking by research nurses (see also online supplementary file S4). There are minor differences in the characteristics of cancer registries of participating jurisdictions, but all are population-based registers with strict monitoring of data quality.

There is some variation in methods of approaching patients, PCPs and CTSs between jurisdictions (see online supplementary file S4). These differences arise from ethical constraints and variations in organisational procedures. In some jurisdictions (such as England), patients needed to be approached by their PCP rather than a direct approach through cancer registries or directly from the local research team. Jurisdictions also differ in the ways they identify patient's PCPs and CTSs, and in the ways they are following up non-responders to the questionnaires.

Registries and clinical databases are used in jurisdictions where questionnaire-based data collection from primary and secondary care was not feasible. This is the case, for example, in Denmark and Northern Ireland data for the hospital component of the pathway. A summary of identification and recruitment processes for all jurisdictions is shown in online supplementary file S4.

\section{Registry data}

From each jurisdiction, available relevant registry data are also collected. These data concern date of diagnosis, Tumour, Node, Metastases (TNM), Fédération
Internationale de Gynécologie et d'Obstétrique (FIGO) and Dukes stage, possible date of death or vital status 12 months after date of diagnosis and screen detected cancer. These data are collected for two purposes: to describe all identified patients with cancer and describe non-participation, and also to do additional analyses on association with survival and mortality.

\section{Sample size}

Sample size considerations are based on the analysis of longer time intervals (more than the 75th centile) across 10 jurisdictions. A reference point is chosen as the jurisdiction, where most patients are expected to have the longest time interval (defined as the largest 75th centile). The proportion with the 'short' interval from the reference jurisdiction will be compared with the proportions with 'short' intervals from the rest of the jurisdictions- that is, nine comparisons will be performed. Our aim is to be able to demonstrate a minimum higher proportion of patients with such 'short' intervals of $85 \%$ (ie, an expected minimal important difference of $10 \%$ ). The sample size calculation is based on sample size determination for comparing proportions by $\chi^{2}$ test in contingency tables. ${ }^{38}$ We have adjusted the method to accommodate our intention to undertake only nine comparisons. With a power of $90 \%$, the method reveals a requirement for an overall sample of size 2000— that is, 200 patients are required in each of 10 jurisdictions.

\section{Data handling}

ICBPM4 data collection is coordinated through the team at UCL, UK, for seven of the jurisdictions. The questionnaire data are entered locally by each of the 10 participating jurisdictions. Seven use a bespoke online data management system (using the Microsoft SQL Server), built by the team at UCL and tested during the English pilot. The system incorporates relevant validation rules for entry of demographic and questionnaire details. The data entered are then transferred without personal identifiers to the central data repository at UCL. Data entry in Victoria (Australia), Sweden and Denmark is via a locally developed database delivered directly to the analysis team in Denmark.

All jurisdictions have established mechanisms to ensure data completeness and quality.

Data are forwarded to the team at University of Aarhus, Denmark, for analysis. Consistent rules for interpreting data are applied to resolve any issues associated with 'incorrect' responses and missing data. These checks are applied consistently to data across all jurisdictions and inconsistencies are discussed with the lead in each jurisdiction. For specific variables on education, ethnicity, comorbidity and smoking, specific standard rules are applied to make the final categorisation of these variables comparable across countries.

\section{Analysis plan}

The primary outcomes of the study are time intervals and routes to diagnosis. A comparison of key diagnostic 
intervals forms the core of our analyses. Since the time intervals will be highly right-skewed, they will be measured with median, interquartiles and 90-centiles. For time intervals, the adjustment will be performed by quantile regression as this method allows for a comparison across the whole distribution of length of the interval. Since the length of the interval in days is a continuous measure which has been rounded, we will use the 'qcount'-routine proposed by Miranda. ${ }^{39}$ One thousand jittered samples will be applied to calculate the parameters of the model.

Where relevant, diagnostic routes will be stratified into 'screening' and 'non-screening'. Adjustment will be carried out with generalised linear models for the binomial family to quantify the prevalence ratio for screening route among jurisdictions. Prevalence ratio (PR) is chosen as an outcome measure over OR, as PR can be more readily understood and communicated-and OR may overemphasise the likelihood when the prevalence of the outcome measure is above $20 \% .^{40}$

Further, we will compare the number of health-related visits and investigations, which is the secondary outcome of the study. Numbers of health-related visits and investigations are considered as counts and will be compared across jurisdictions by using negative binomial regression. We plan to use negative binomial distribution to ensure that potential non-equality of conditional mean and conditional variance in the data is dealt with appropriately. ${ }^{41}$

Univariable and multivariable models will be used. The multivariable analyses will control for patient-related factors such as sex, age, comorbidity, education and whether a patient had cancer-specific symptoms.

Data quality analyses will be performed to identify missing, incomplete, 'out of range' and inconsistent data. This will include testing for negative (or very large) time intervals and multiple responses (ie, cases where there are two dates given in the same survey). We have developed rules (see below) for handling conflicting responses (eg, a respondent indicating that he/she is both a smoker and non-smoker).

We are using three different sources of data on patient journeys. Therefore, a comprehensive set of data rules has been developed based on previous analyses in the UK and Denmark. For some questions, we will have responses from the patient and both their PCP and CTS. Hence, for each question, there is a 'hierarchy' of responses and we have decided a priori which source (patient, PCP or CTS questionnaire) should take priority. For example, we will preferentially use patient data for dates of first symptoms, and hospital data for diagnostic information, while for 'date of first presentation' we are giving preference to PCP responses. We also prioritise a specific date higher than a less specific or a categorised time interval. The exact data rules for each cancer site will be published in the subsequent ICBPM4 papers.

We will report on validity and reliability between our three data sources. This triangulation analysis will focus on comparing items such as dates, intervals and routes to diagnosis. The agreement for categorical variables will be assessed by $\kappa$ coefficients. The agreement for continuous variables will be measured by Lin's concordance correlation coefficient. ${ }^{42}$ If necessary, we will perform transformation to account for the skewed data. Validation by measuring against a gold standard will be performed in those jurisdictions where data from a registry are available.

Statistical analyses will be carried out using STATA V.14 software.

\section{Ethical and other approvals}

A list of the ethical, privacy and data approvals that we received is shown in online supplementary file S5. All jurisdictions have strict governance and privacy regulations for use of cancer registry data-in particular to ensure that confidentiality of patient information is not compromised.

\section{Preliminary response rates}

Online supplementary file S6 provides a summary of progress with patient and primary care recruitment across all jurisdictions up to the end of February 2016. These preliminary data illustrate the degree to which the recruitment strategy has been successful. An important factor has been the time it takes from diagnosis to registration in our participating registries (it ranges from $\sim 1$ to 4 months), given that our recruitment window is 3-6 months postdiagnosis - this window is based on avoiding recruitment either too soon after diagnosis (when treatment may be active), or too long-in which case recollection of prediagnosis events might be diminished.

In UK countries, patient questionnaire response rates until now are typically highest for breast cancer, and lowest in lung cancer. Recruitment in Northern Ireland was by direct approach, and this has achieved higher overall response rates than the other countries of the UK-although if only rates amongt questionnaires forwarded by PCPs in the UK are considered, the rates are more similar. Denmark achieved higher response rates than UK countries-the reasons for this are unclear, and may reflect cultural and social differences which influence willingness to respond to surveys. Responses from primary care are consistently higher than patient responses so far in all jurisdictions.

\section{Assessing non-response and study validity}

All jurisdictions are collecting standardised data on all essential figures to describe internal validity and study flow. During the study period all patients with cancer were identified. Information on total eligible patients, exclusions (and reasons for exclusion) and numbers declining to participate (with their reasons) was also collected. This will form the basis for an analysis of selection bias and validity of the study in terms of drawing comparisons between countries. 


\section{DISCUSSION}

ICBPM4 is the first attempt we are aware of to describe and compare, between countries, patient journeys to a cancer diagnosis and treatment. While there has been some preliminary work examining the logistics of such comparisons, ${ }^{43} 44$ there are no previous examples of applying standardised survey methods in a broad range of international jurisdictions to systematically examine the various components of diagnostic intervals. It is a complex exercise with a broad range of methodological challenges; nevertheless, it has the potential to highlight important differences in diagnostic routes and these may contribute to an understanding of variations in cancer survival.

In international studies, it is important that data collection is standardised as much as possible across jurisdictions while accommodating differences in language and cultural understanding of health-related terms. We have chosen to use self-completion surveys for our data collection (except for secondary care data in Northern Ireland and Denmark where collection from healthcare records was considered more feasible). There are further slight variations in the administration of the questionnaire across jurisdictions (as described in online supplementary file S4). Our questionnaire development drew on existing instruments, and went through an extensive process of cognitive testing, piloting and translation/harmonisation. Nevertheless, the small number of patients we used in the test-retest analysis means that our reliability estimates have a low level of statistical precision.

We have been able to present preliminary response rate data; our rates are similar to a Danish study which used registry-based recruitment, and sample both patients and PCPs (in which response rates were $79 \%$ for PCPs and $40 \%$ for patients; ${ }^{42}$ while PCPs have much lower response rates to general surveys, enquiry about specific patients (as is the case in ICBPM4) appears to lead to a significantly higher response. One of our principal concerns is dealing with non-response and, in particular, differential response rates across jurisdictions.

Response to a survey on diagnostic journeys may be influenced by several factors, including levels of comorbidity, awareness of cancer symptoms, healthseeking behaviour and participation in screeningfurther, very ill patients may be under-represented due to their circumstances-and their shorter survival. Reasons for non-response may differ between jurisdictions, and differential response rates may compound any resulting bias. Accordingly, our analyses will include a careful examination of sociodemographic characteristics, and non-response bias will be explored as thoroughly as possible with available data. All results will be carefully scrutinised in the light of non-response issues and, where possible, adjustments will be made-along with clearly expressed caveats over the interpretation of our results. There are further patient-specific factors which ICBPM4 methods need to accommodate, including examining the implications of previous or synchronous cancers on self-reports of cancer journeys.

\section{CONCLUSION}

An international questionnaire-based survey (ICBPM4) of patients, PCPs and hospital specialists has been developed and launched in 10 international jurisdictions. In this paper, we have reported on key aspects of survey design, questionnaire development and testing and initial response rates.

ICBPM4 will form the basis for several analyses on aspects of routes to cancer diagnosis and time intervals. It will take into account a broad range of methodological limitations, and appropriate caveats will be applied in the interpretation of results. In combination with other ICBP modules, ICBPM4 has significant potential to help us increase the understanding of international differences in cancer survival. Combining ICBPM4 data with data and results from other ICBP modules will enable analyses to be undertaken on the association between healthcare organisation, access to investigation and public awareness of cancer symptoms and time and routes to diagnosis. This has the potential to inform future interventions aimed at reducing diagnostic intervals relating to patients, their healthcare providers and systems and thereby improving outcomes.

Author affiliations

${ }^{1}$ Centre for Population Health Sciences, University of Edinburgh, Edinburgh, UK

${ }^{2}$ Department of Public Health, Research Unit for General Practice, Aarhus University, Aarhus, Denmark

${ }^{3}$ Gynaecological Cancer Research Centre, Women's Cancer, Institute for Women's Health, University College London, London, UK

${ }^{4}$ Department of Prevention and Cancer Control, Cancer Care Ontario, Toronto, Ontario, Canada

${ }^{5}$ Centre for Public Health, Queen's University Belfast, Northern Ireland Cancer Registry, Belfast, UK

${ }^{6}$ Department of Family and Community Medicine, Knowledge Translation Research Network Health Services Research Program, Ontario Institute for Cancer Research, University of Toronto, Toronto, Ontario, Canada

${ }^{7}$ Department of Medical Epidemiology and Biostatics, Regional Cancer Center Uppsala and, Karolinska Institutet, Stockholm, Sweden

${ }^{8}$ North Wales Centre for Primary Care Research, Bangor University, Wrexham, UK

${ }^{9}$ Department of Oncology, Lund University Hospital, Lund, Sweden

${ }^{10}$ Population Oncology, CancerCare Manitoba, Winnipeg, Manitoba, Canada

${ }^{11}$ Centre for Behavioral Research in Cancer, Cancer Council Victoria,

Melbourne, Victoria, Australia

${ }^{12}$ Department of Policy and Information, Cancer Research UK, London, UK

Acknowledgements Catherine Foot, Martine Bomb, Brad Groves and Samantha Harrison of Cancer Research UK for managing the programme. William Liston at University College London for his work in building and supporting the database for use by participating jurisdictions. The ICBPM4 academic reference group for providing independent peer review and advice for the study protocol and analysis plan development. the authors would like to thank all patient groups who took part in the testing and validation of our study materials, and all patients, health care providers and Clinical Research Nurses (Northern Ireland) who participated in our pilot studies for taking the time to contribute to this international study. The ICBP Programme Board: Ole Andersen (Danish Health and Medicines Authority, Copenhagen, Denmark), Søren Brostrøm (Danish Health and Medicines Authority, Copenhagen, Denmark), Heather Bryant (Canadian Partnership Against Cancer, Toronto, Canada), David Currow (Cancer Institute New South Wales, Sydney, Australia), Dhali Dhaliwal (CancerCare Manitoba, Winnipeg, Canada), Anna Gavin (Northern Ireland Cancer Registry, Queens University, Belfast, UK), Gunilla 
Gunnarsson (Swedish Association of Local Authorities and Regions, Stockholm, Sweden), Jane Hanson (Welsh Cancer National Specialist Advisory Group, Cardiff, UK), Nicola Quin (Cancer Council Victoria, Carlton, Australia), Stein Kaasa (Norwegian University of Science and Technology, Trondheim, Norway), Linda Rabeneck (Cancer Care Ontario, Toronto, Canada), Michael A Richards (Care Quality Commission, London, UK), Michael Sherar (Cancer Care Ontario, Toronto, Canada), Robert Thomas (Department of Health and Human Services, Victoria, Melbourne, Australia).

Collaborators ${ }^{*}$ Online supplementary file 5 contains a list of the full ICBP Module 4 Working Group.

Contributors DW, PV and UM are lead investigators on ICBPM4 and produced first versions of this manuscript. CA, AZ, EOF, RD, WL, HJ and MB are involved in the central co-ordination of ICBPM4, and made significant contributions to the paper, including the provision of technical information. AG, EG, ML, R-JL, MM, RDN, JK, DT and VW have lead roles in their individual jurisdictions, and also contributed substantially to the paper, particularly from the perspective of their own jurisdictions. The ICBPM4 working group contributed to the study through data collection, technical support and other activities essential to the production of this paper. Every author takes responsibility for the entire content of the manuscript.

Funding CancerCare Manitoba; Cancer Care Ontario; Cancer Council Victoria; Cancer Research Wales; Danish Cancer Society; Danish Health and Medicines Authority; European Palliative Care Research Centre (PRC), Norwegian University of Science and Technology (NTNU); Guidelines Audit and Implementation Network (GAIN); Macmillan Cancer Support; National Cancer Action Team; NHS England; Northern Ireland Cancer Registry, funded by the Public Health Agency Nl; Norwegian Directorate of Health; Research Centre for Cancer Diagnosis in Primary Care (CaP), Aarhus University, Denmark; Scottish Government; Swedish Association of Local Authorities and Regions; University College London; University of Edinburgh; Victorian Department of Health and Human Services; Welsh Government.

Competing interests None declared.

Ethics approval Supplementary file 5 contains all ethics approval details.

Provenance and peer review Not commissioned; externally peer reviewed.

Data sharing statement No additional data are available.

Open Access This is an Open Access article distributed in accordance with the Creative Commons Attribution Non Commercial (CC BY-NC 4.0) license, which permits others to distribute, remix, adapt, build upon this work noncommercially, and license their derivative works on different terms, provided the original work is properly cited and the use is non-commercial. See: http:// creativecommons.org/licenses/by-nc/4.0/

\section{REFERENCES}

1. Butler J, Foot C, Bomb M, et al. The International Cance Benchmarking Partnership: an international collaboration to inform cancer policy in Australia, Canada, Denmark, Norway, Sweden and the United Kingdom. Health Policy 2013;112:148-55.

2. Coleman MP, Forman D, Bryant $\mathrm{H}$, et al. Cancer survival in Australia, Canada, Denmark, Norway, Sweden, and the UK, 19952007 (the International Cancer Benchmarking Partnership): an analysis of population-based cancer registry data. Lancet 2011;377:127-38.

3. De Angelis R, Sant M, Coleman MP, et al. Cancer survival in Europe 1999-2007 by country and age: results of EUROCARE-5-a population-based study. Lancet Oncol 2014;15:23-34.

4. Walters S, Maringe C, Butler J, et al. Breast cancer survival and stage at diagnosis in Australia, Canada, Denmark, Norway, Sweden and the UK, 2000-2007: a population-based study. Br J Cancer 2013;108:1195-208. ISSN 0007-0920

5. Forbes LJ, Simon AE, Warburton F, et al. Differences in cancer awareness and beliefs between Australia, Canada, Denmark Norway, Sweden and the UK (the International Cancer Benchmarking Partnership): do they contribute to differences in cancer survival? Br J Cancer 2013;108:292-300.

6. Rose PW, Rubin G, Perera-Salazar R, et al. Explaining variation in cancer survival between 11 jurisdictions in the International Cancer
Benchmarking Partnership: a primary care vignette survey. BMJ Open 2015;5:e007212.

7. Allemani $\mathrm{C}$, Weir HK, Carreira $\mathrm{H}$, et al. Global surveillance of cancer survival 1995-2009: analysis of individual data for 25,676,887 patients from 279 population-based registries in 67 countries (CONCORD-2). Lancet 2015;385:977-1010.

8. Mitchell E, Macdonald S, Campbell NC et al Influences on pre-hospital delay in the diagnosis of colorectal cancer: a systematic review. Br J Cancer 2008;98:60-70.

9. Tørring ML, Frydenberg M, Hansen RP, et al. Time to diagnosis and mortality in colorectal cancer: a cohort study in primary care. $\mathrm{Br} \mathrm{J}$ Cancer 2011;104:934-40.

10. Tørring ML, Frydenberg M, Hamilton W, et al. Diagnostic interval and mortality in colorectal cancer: U-shaped association demonstrated for three different datasets. J Clin Epidemiol 2012;65:669-78.

11. Tørring ML, Frydenberg M, Hansen RP, et al. Increased mortality among cancer patients with longest diagnostic intervals: a cohort study in primary care. Eur J Cancer 2013;49:2187-98.

12. Neal RD, Tharmanathan $\mathrm{P}$, France $\mathrm{B}$, et al. Increased time to diagnosis and treatment in symptomatic cancer associated with poorer outcomes? Systematic review. Br J Cancer 2015;112 (Suppl 1):S92-107.

13. Elliss-Brookes L, McPhail S, Ives A, et al. Routes to diagnosis for cancer-determining the patient journey using multiple routine data sets. Br J Cancer 2012;107:1220-6.

14. Black G, Sheringham J, Spencer-Hughes V, et al. Patients' experiences of cancer diagnosis as a result of an emergency presentation: a qualitative study. PLOS ONE. 2015;10: e0135027.

15. Cleary J, Peters TJ, Sharp D, et al. Clinical features of colorectal cancer before emergency presentation: a population-based casecontrol study. Fam Pract 2007;24:3-6.

16. Booij JC, Zegers M, Evers PM, et al. Improving cancer patient care: development of a generic cancer consumer quality index questionnaire for cancer patients. BMC Cancer 2013;13:203.

17. Larsen MB, Hansen RP, Olesen F, et al. Patients' confidence in their GP before and after being diagnosed with cancer. Br J Gen Pract 2011;61:e215-22.

18. Smith EC, Ziogas A, Anton-Culver H. Delay in surgical treatment and survival after breast cancer diagnosis in young women by race/ ethnicity. JAMA Surg 2013;148:516--23.

19. Department of Health. Direct access to diagnostic tests for cancer. Best practice referral pathways for general practitioners, 2012 https://www.gov.uk/government/publications/direct-access-todiagnostic-tests-for-cancer-best-practice-referralpathways-for-general-practitioners (accessed Mar 2015).

20. Hamilton W, Green T, Martins T, et al. Evaluation of risk assessment tools for suspected cancer in general practice: a cohort study. $\mathrm{Br} \mathrm{J}$ Gen Pract 2013;63:e30-6.

21. Vedsted $\mathrm{P}$, Olesen F. Are the serious problems in cancer survival partly rooted in gatekeeper principles? An ecologic study. $\mathrm{Br} J \mathrm{Gen}$ Pract 2011;61:e508-12.

22. Walters S, Maringe C, Coleman MP, et al. Lung cancer survival and stage at diagnosis in Australia, Canada, Denmark, Norway, Sweden and the UK: a population-based study, 2004-2007. Thorax 2013;68:551-64. ISSN 0040-6376

23. Maringe $\mathrm{C}$, Walters $\mathrm{S}$, Rachet $\mathrm{B}$, et al. Stage at diagnosis and colorectal cancer survival in six high-income countries: a population-based study of patients diagnosed during 2000-2007. Acta oncol 2013;52:919-32. ISSN 0284-186X.

24. Wang J, Mahasittiwat $\mathrm{P}$, Wong KK, et al. Natural growth and disease progression of non-small cell lung cancer evaluated with 18F-fluorodeoxyglucose PET/CT. Lung Cancer 2012;78: 51-6.

25. Jensen AR, Nellemann HM, Overgaard J. Tumor progression in waiting time for radiotherapy in head and neck cancer. Radiother Oncol 2007;84:5-10.

26. Maringe $\mathrm{C}$, Walters $\mathrm{S}$, Butler J, et al. Stage at diagnosis and ovarian cancer survival: evidence from the International Cancer Benchmarking Partnership. Gynecol Oncol 2012;127:75-82.

27. The International Benchmarking Partnership: ICBPM4. http://www. cancerresearchuk.org/cancer-info/spotcancerearly/ICBP/module-4/ (accessed Mar 2015)

28. Weller D, Vedsted P, Rubin G, et al. The Aarhus statement: improving design and reporting of studies on early cancer diagnosis. Br J Cancer 2012;106:1262-7.

29. Burgess C, Hunter MS, Ramirez AJ. A qualitative study of delay among women reporting symptoms of breast cancer. Br J Gen Pract 2001;51:967-71. 
30. Larsen MB, Hansen RP, Sokolowski I, et al. Agreement between patient-reported and doctor-reported patient intervals and date of first symptom presentation in cancer diagnosis-a population-based questionnaire study. Cancer Epidemiol 2014;38:100-5.

31. Lyratzopoulos G, Abel GA, McPhail S, et al. Measures of promptness of cancer diagnosis in primary care: secondary analysis of national audit data on patients with 18 common and rarer cancers. Br J Cancer 2013;108:686-90.

32. Hansen RP, Vedsted P, Sokolowski I, et al. Time intervals from first symptom to treatment of cancer: a cohort study of 2,212 newly diagnosed cancer patients. BMC Health Serv Res 2011;11:284.

33. Neal RD, Pasterfield D, Wilkinson C, et al. Determining patient and primary care delay in the diagnosis of cancer-lessons from a pilot study of patients referred for suspected cancer. BMC Fam Pract 2008;9:9.

34. Jobe JB, Mingay DJ. Cognitive research improves questionnaires. Am J Public Health 1989;79:1053-5.

35. IARC. In: Forman D, Bray F, Brewster DH, Gombe Mbalawa C, Kohler B, Piñeros M, Steliarova-Foucher E, Swaminathan R, Ferlay J, (eds). Cancer Incidence in Five Continents, Volume X. IARC Scientific Publications No. 164. International Agency for Research on Cancer Lyon, France: International Agency for Research on Cancer. 2014. https://www.iarc.fr/en/publications/pdfsonline/epi/sp164/CI5volX_Full.pdf
36. Bray F, Parkin DM. Evaluation of data quality in the cancer registry: principles and methods. Part I: comparability, validity and timeliness. Eur J Cancer 2009;45:747-55.

37. Parkin DM. The evolution of the population-based cancer registry. Nat Rev Cancer 2006;6:603-12.

38. Agresti A. Frontmatter. In: Categorical data analysis. 2nd edn Hoboken, NJ, USA: John Wiley \& Sons, Inc, 2002. doi: 10.1002/ 0471249688.

39. Miranda A. QCOUNT: Stata program to fit quantile regression models for count data. Boston: Boston College Department of Economics, 2006

40. Barros AJ, Hirakata VN. Alternatives for logistic regression in crosssectional studies: an empirical comparison of models that directly estimate the prevalence ratio. BMC Med Res Methodol 2003;3:21.

41. Cameron AC, Trivedi PK. Regression analysis of count data. 2nd edn. Econometric Society Monograph No. 53, Cambridge University Press. 2013.

42. Lin LI. A concordance correlation coefficient to evaluate reproducibility. Biometrics 1989;45(1):255-68.

43. Murchie $\mathrm{P}$, Campbell NC, Delaney EK, et al. Comparing diagnostic delay in cancer: a cross-sectional study in three European countries with primary care-led healthcare systems. Fam Pract 2012;29:69-78.

44. Jensen $\mathrm{H}$, Tørring ML, Larsen MB, et al. Existing data sources for clinical epidemiology: Danish cancer in primary care cohort. Clin Epidemiol 2014;6:237-46. 